\title{
Research on the Establishment of Competency Model of Teachers in Universities
}

\author{
Fang Wang ${ }^{1, a}$ \\ ${ }^{1}$ Business School, Xi'an Peihua University, Xi'an, 710000, China \\ ${ }^{\mathrm{a}}$ email
}

Keywords: Competency model, Model establishment, Teachers in universities

\begin{abstract}
The theory and research results related to competency model are becoming more and more important in the field of human resource management of teachers in universities. This paper combs the significance, principles and methods of the construction of competency model of university teachers. At the same time, this paper gives the specific processes of the construction of competency model of teachers in universities, including the processes of questionnaire design, indexes selection and weights identification methods to provide some references for the relevant researchers.
\end{abstract}

\section{Introduction}

Competency model refers to the sum of competency required for a specific task role. It's a set of competencies that are specific to specific job performance requirements. The competency model provides a successful model for a specific organization, level, job or role, reflect the influence of individual behavior, all the important skills and knowledge in a given job, which is often cited as a tool for use in the workplace. With competency becoming the research focus of management psychology, its research results have gradually penetrated into the practice of human resource management and development. In competency based human resource management, competency model is the basis of human resources management functions. Individual competency model can help to distinguish the competence, the advantage of work, the need to improve the field of improvement, continuing learning and career growth and development. Teachers' competency, which belongs to the individual characteristics of teachers, is a necessary condition for teachers to engage in successful teaching and the main training objectives of teachers' educational institutions. At present, the academic circles at home and abroad on Teachers' competency are no standard definition. Some scholars at home and abroad have in common that the definition of teachers' competence should include successful teaching related professional knowledge and professional skills and implementation. But in other competency characteristics is controversial: foreign scholars believe that teachers' personality to show competency; domestic scholars believe that the professional teacher's values, namely personal morality, occupation morality to show competency. From the above definition of scholars, this paper adopts the teacher competency definition: teacher competency and job performance in teaching is closely related, and can be generalized to describe the behavior patterns of individual teachers is relatively fixed and lasting characteristics.

\section{Significance, Principles and Methods of Establishment of Competency Model of Teachers in Universities}

Significance. As a social organization, colleges and universities bear the important task of personnel training, knowledge innovation and social services. The teacher resources as the first resource in Colleges and universities, the overall quality of teachers has a major impact on the survival and development of colleges and universities. In recent years, society has put forward higher requirements for the quality and efficiency of teachers' work. To establish the competency model of teachers in Colleges and universities, is conducive to a comprehensive description of high performance characteristics of teachers, the university system of recruitment, selection, training, personnel evaluation, improve the overall quality of teachers, enhance the core competitiveness of the 
school will have greater significance. Although the competency research method in the world-wide impact and research upsurge, and obtained a lot of achievements, but the existing research mostly around the enterprise leadership competency, especially domestic research in the field of education, especially the empirical research on university teachers competency is relatively limited. At the same time, with the deepening of economic development and personnel system reform, many researchers regard competency evaluation as an effective means to evaluate job performance. In addition to the development of theoretical models, a large number of literatures have also studied the relationship between competency and performance variables, although most of the research results show that competency can improve the prediction of organizational performance. In other words, competency has a positive correlation with various performance variables. However, the research on Chinese universities is relatively deficient. Therefore, this paper intends to start from the Chinese college background, the relationship between the structure of competency model of teachers in Colleges and universities in China and its relationship with the performance of college teachers, so as to expand the study of China's University Teachers' competency model, and will provide a new perspective for the research of this field, and lay a solid foundation for further study in the future.

Principles. The competency evaluation system of university teachers should first be operable. It is practical and practical. It can be widely used and truly become a useful tool for teacher selection, evaluation, promotion, assessment and training in colleges and universities. In order to give full play to the teacher's occupation ability of growth oriented performance evaluation of teachers' teaching process and result oriented combination, especially need to pay attention to process evaluation, comprehensive quality and performance of the teachers' attention and guidance and comprehensive evaluation. Scientific principle is one of the basic principles of educational evaluation system. The evaluation process and all its main directions should be in line with the objective laws of the development of education itself. The evaluation system of university teachers needs to embody the principle of science, which can guarantee the rationality of the practical application and management practice of education and teaching, and meet the needs of practical teaching management to the maximum extent. The evaluation system of university teachers' competency needs scientific evaluation of various evaluation indicators, so as to carry out a measurable and verifiable integrity system. The index system of competency is a set of tree structure index, which is composed of a plurality of subsets, establish the evaluation system of university teachers, teachers can not only external evaluation, but also for the internal index and weight index may also have internal measurement and assessment objective, standard and system.

Methods. To establish the competency model of teachers is a prerequisite for human resources management of university teachers, University Teachers competency through constructing the content system, can provide the basis for the evaluation of competency evaluation for university teachers competency assessment, selection and training. The commonly used methods for the construction of competency model for college teachers include the following BEI methods: behavioral event interview. The main steps of the method include: first, establishment and development of universities should be consistent with the identification standard, refine the evaluation standard of high performance and general performance of teachers, such as the level of published papers and the number; secondly, according to the identification criteria, the general performance of high performance, a certain amount of teachers were selected as samples and according to the evaluation standards, interviews of two groups of samples by using behavioral event interview; thirdly, the sample collection and relevant data, to determine who can distinguish the competency; then, data analysis, model; finally, re select the test samples. In the process of establishing the model with this method, researchers should ensure the reliability and validity of the interview and coding. Therefore, the personnel who interview and encode should have certain professional knowledge and objectivity. Analytic hierarchy process. The index system of this method can be divided into three layers: general target, first level and two-level index. To calculate and determine the weight factor of each level using a specific mathematical method, in order to reflect the influence of each index value of comprehensive index, and according to the measurement value to analyze and determine the competency model of grey decision-making based on grey system theory, 
the correlation degree, the correlation coefficient of two quantitative indicators from the whole or dynamic measure or correlation and quantitative analysis of things between factors, to provide quantitative basis for the main factor of formation of accurate identification of dynamic changes of things.

\section{Specific Processes of Establishment of Competency Model of Teachers in Universities}

Design of Questionnaire. In the interview, respondents talking about success and failure of their events, often related to the objective factors, such as the relationship between teachers and students, school management and so on, and the key for the teaching and scientific research work they are engaged in less involved. Therefore, only from teachers to construct the competency model have certain defects, also should consider the teaching object itself is students' perceptions of competency model, this study adopts questionnaire survey method to explore the perspective of students' teachers. Prior to the interview, respondents were contacted to inform respondents of the purpose of the study and to determine the time and place of the interview. And give the interview outline in advance so that the interviewee is ready. Formal interviews were completed after all the preparations were completed. In the interview process, according to the description of the interviewee, ask various detection problems, in-depth detection of events. Each interviewee's interview time was controlled in about one hour. Interview recording. Based on the above interview data and previous literature, can find the competency of teachers in private colleges should include the following dimensions: the features of research, such as research consciousness and research ability, planning ability, information acquisition ability analysis. Teaching characteristics, such as classes, instructive language, learning ability, class interaction; personality characteristics, such as self-confidence, tolerance, initiative, personality; knowledge characteristics, such as professional knowledge and skills, enrich the reasonable knowledge structure, advanced education idea, knowledge structure with service; the characteristics, such as respect for others, team spirit, good interpersonal relationship, keen observation.

Selection of Indexes. Different scholars, in the definition of teacher competency and competency model, exists, the contention of a hundred schools of thought each one airs his own views. Teachers' competency refers to the school teaching work, the difference factors and the performance of good teachers and mediocre teachers, the main features of the two aspects, internal features and external characteristics. According to the work of teachers, and combined with the current research results of all scholars, can be the index of competency of Teachers in Colleges and universities is divided into four aspects. The first one is the overall quality of knowledge culture; second is the overall quality of technical ability; third is the attitude and view of value, the overall quality of the overall quality; the fourth is the driving force of personality and work. These four indicators the basic competency includes three aspects: the cultural knowledge and technical ability in the outer performance through the external form; attitude and values in the middle, personality and work force in the innermost layer, but also the most important, the most critical layer. Knowledge quality includes professional theory knowledge, professional practice knowledge and educational theory knowledge. The professional theory knowledge is the teacher's educational background, professional title, teaching experience, and the accumulation of professional theoretical knowledge of the subject or course. Professional practice knowledge is the accumulation of practice and application knowledge of this discipline or curriculum, and the education theory knowledge is the accumulation of theoretical knowledge of education management and education psychology. Emotional characteristics include tolerance, emotional stability and frustration tolerance. Tolerance refers to the students' problems, bad behavior can tolerate, but not indulgence students. To maintain emotional stability, free from arrogance and impetuosity emotions be neither humble nor pushy. Frustration tolerance refers to the rational view of setbacks, setbacks, not giving up, uncompromising attitude. Personality traits include self-identity, self-motivation and teaching efficacy. Self-identity refers to the identity of teachers in colleges.

Identification of Weights. Analytic hierarchy processes a method of decomposing order from total to total. It calculates the weight proportion of each factor in each level by solving the vector of 
the characteristic of judgment matrix. The analytic hierarchy processes more complex and more complex. It is suitable for complex index system and hierarchical target object research. Its main way is to construct a judgment matrix, and then calculate the factors that occupy the largest eigenvalue. After the corresponding eigenvector is normalized, it is the value of the relative importance weight of the upper level index at a hierarchical index. Analysis of related literatures and combined with the result of domestic and foreign scholars present research results and research, in the calculation of University Teachers' evaluation index weight, first need to first consider the importance of the four level indexes, and according to the importance of the four indicators of the order. Self-evaluation highlights the dominant position of teachers in evaluation, and helps teachers to reflect on themselves, so as to conduct a qualitative evaluation of teachers' work attitude, personality quality, knowledge and skills, and behavior of performing their duties. The premise is that the academic community at different levels to review it, and issued by the relevant academic representatives of the final evaluation conclusions. Although the teaching evaluation for different purposes, but the value orientation of reasonable teaching evaluation should become the common requirements of various evaluation subject value selection, and this common choice required by the academic community exercise evaluation responsibilities, grasp the right words, which is widely recognized, to carry out systematic evaluation of teaching. The four indicators are the four important indicators to constitute the competency of university teachers, but the degree of importance is different. According to the iceberg theory and other relevant competency models, the importance of the four indicators should increase progressively, and the latter is higher than the former. The first important should be personality and work driving force, followed by attitude, values, third is technical ability, and finally for knowledge culture. In this, the first professional knowledge level is the most important one, second is to master the professional development of advanced information and knowledge, relatively speaking, master the basic theory of education knowledge is the most weakening. The two indicators of college teachers' personality and work drive evaluation are physical and mental health, achievement motivation, responsibility, and the two levels of evaluation of college Teachers' personality and driving force.

\section{Conclusion}

In this study, through literature collection, analytic hierarchy process and other methods, initially established the evaluation system of university teachers' competency. Although this paper has formed an evaluation system for the competency of university teachers in our country, the amount of calculation is huge because of the various evaluation indexes involved. The actual situation of different colleges and universities is not consistent, so it is suggested that the relevant evaluation indicators need to be adjusted and changed under different circumstances.

\section{References}

[1] Fu Min, Zhang Lulu. A review of Chinese competency model research of teachers in Colleges and Universities [J]. Teacher Education Forum, 2014, 27(9): 24-27.

[2] Yan Zhengshu. Research on the Construction of MOOC Teaching Competency Model of College Teachers [J]. Open Education Research, 2015, 21(6): 104-111.

[3] Cao Rong, Xu Shunfeng, Lin Su, et al. Constructing the Teaching Competency Model of Young Teachers in Colleges and Universities [J]. Chinese Journal of Ergonomics2016, 22(1): 44-49+56.

[4] Huang Lan, Fan Xiaojie. On Teaching Evaluation of College Teachers Based on Competency Model [J]. Modern Education Science, 2017(2): 71-75. 